\title{
SIMPLICIDADE NA CIÊNCIA
}

\author{
J. Lima-de-Faria* \\ Instituto de Investigação Científica Tropical \\ jlimadefaria@hotmail.com
}

\begin{abstract}
Simplicity in Science - A short analysis of the simplicity in Science is presented, and a crystallographic example of this simplicity is given as the description of crystal structures of general formulas $A B, A B_{2}, A B_{3}, A B_{4}$, based on cubic or hexagonal close packings of the large atoms, with the small atoms occupying the octahedral or tetrahedral interstices, after an appropriate interpretation and representation by condensed models.

É feita uma breve análise sobre a simplicidade na Ciência, e é apresentado um exemplo dessa simplicidade em Cristalografia, num quadro de estruturas cristalinas de fórmulas gerais $\mathrm{AB}, \mathrm{AB}_{2}, \mathrm{AB}_{3}, \mathrm{AB}_{4}$, baseadas em empilhamentos densos, cúbico ou hexagonal dos átomos maiores, com os átomos pequenos ocupando os interstícios octaédricos ou tetraédricos. Uma interpretação adequada e a representação por meio de modelos condensados dessas estruturas, põem em evidência a semelhança e simplicidade dessas estruturas.
\end{abstract}

É espantosa a enorme diversidade da Natureza, mas também é igualmente espantoso que, em muitos casos, as regras e os mecanismos que a governam sejam muito simples. Paul Dirac foi um dos cientistas mais interessados pela simplicidade e beleza da ciência, e escreveu: "Na sua essência a Natureza é simples” [1].

A simplicidade da Ciência é uma consequência da simplicidade escondida da Natureza. Para Einstein "o que é mais incompreensível no nosso Universo é ele ser compreensível” [2]. Esta simplicidade da Ciência é possivelmente parte da resposta à perplexidade de Einstein.

Na Física, na Química, e na Cristalografia, assim como noutros ramos da ciência, encontramos muitas vezes essa simplicidade. Na Física as leis são normalmente representadas por expressões matemáticas simples. No que diz respeito à Química podemos recordar uma das suas leis fundamentais, a lei das proporções múltiplas, que estabelece: "quando dois elementos se combinam para formar mais do que um composto, os pesos de um elemento que se combina com o mesmo peso do outro estão na razão de números inteiros e pequenos”. Esta lei foi estabelecida por John Dalton, cerca de 1803, e resultou largamente da sua especulação sobre as bases da teoria atómica, que veio a simplificar imenso a ciência da química.

Na Cristalografia, uma das leis fundamentais é a lei de Haüy, que estabelece que: "se escolhermos três faces num cristal como planos de coordenadas, e uma quarta face como unidade, os índices de todas as faces e as arestas são números inteiros, e usualmente pequenos". É curioso notar um certo paralelismo entre a lei química de Dalton e a lei cristalográfica de Haüy.

Como cristalógrafo vou tratar agora, com mais detalhe, alguns aspectos da cristalografia. Em cristalografia, para des-

* Investigador Coordenador Jubilado crever as estruturas cristalinas, existem duas ferramentas fundamentais: a representação das estruturas por meio de modelos atómicos e o uso de fórmulas estruturais. A representação das estruturas pode ser feita de várias maneiras: por meio de modelos de bolas e pequenas hastes que as ligam, pela projecção dos átomos num dos lados da malha unitária; pela distribuição dos átomos em perspectiva na malha unitária, pela descrição da coordenação poliédrica dos átomos, e pelo empilhamento atómico. Uma outra maneira de representação é por sobreposição de camadas de átomos, os chamados modelos condensados [3].

A maior parte das estruturas cristalinas podem ser descritas pela sobreposição de camadas de átomos todas iguais, ou por duas diferentes que alternam. As camadas são representadas por folhas de acetato transparente, e os átomos desenhados por círculos de tamanhos diferentes. No caso de estruturas baseadas em empilhamentos densos dos átomos maiores, com átomos mais pequenos ocupando os interstícios desses empilhamentos, foram preparadas folhas padrão com todos os interstícios desenhados (quer octaédricos quer tetraédricos), e onde é fácil marcar os interstícios que estão ocupados. As camadas transparentes padrão colocam-se num suporte apropriado para permitir uma visualização 3D da estrutura cristalina.

Paul Moore [4] atribuiu grande importância à representação das estruturas e defendeu que se escolhermos a descrição apropriada descobriremos que muitas estruturas são baseadas em empilhamentos densos que anteriormente não tinham sido descritas como tais [5]. Num artigo recente de J. Lima-de-Faria [6] são descritas 322 estruturas cristalinas baseadas em empilhamentos densos, e mais 135 baseadas noutros tipos de empilhamento, num sentido mais geral.

As fórmulas estruturais que caracterizam as estruturas cristalinas são também muito importantes para a sua compre- 
ensão, e a sua notação deve ser o mais evidente possível. Foram várias as notações que foram propostas para as estruturas cristalinas, mas destas quero destacar a de Nigli [7] e a de Machatschki [8]. Em 1965 J. Lima-de-Faria [9] propôs uma notação para os tipos estruturais inorgânicos, e em 1976, esta foi adaptada por J. Lima-de-Faria e M. O. Figueiredo [10] como fórmulas estruturais das estruturas cristalinas. Mais tarde, estas fórmulas estruturais foram recomendadas pela Comissão Internacional de Nomenclatura da União Internacional de Cristalografia [11].

Vamos agora considerar um conjunto de estruturas cristalinas de compostos de fórmulas gerais $\mathrm{AB}, \mathrm{AB}_{2}, \mathrm{AB}_{3}$ e $\mathrm{AB}_{4}$, e comparar a sua representação convencional com a representação por meio dos modelos condensados.
Se usarmos a sua representação convencional, baseada na distribuição geométrica dos átomos ou através da sua coordenação poliédrica, e se usarmos modelos cristalográficos, tais como os de bolas e hastes, ou formados por poliedros de coordenação, torna-se difícil visualizar o arranjo dos átomos, e as estruturas parecem complexas e todas muito diferentes (Figura 1).

Se, no entanto, fizermos uma interpretação apropriada dessas estruturas e usarmos como representação os modelos condensados, verifica-se que estas estruturas são semelhantes, todas baseadas em empilhamentos densos, formadas por camadas muito simples, todas iguais, ou duas alternadas, com uma distribuição simples e simétrica dos seus átomos (Figura 2).

\begin{tabular}{|c|c|c|c|c|c|}
\hline \multicolumn{2}{|c|}{ AB } & \multicolumn{2}{|c|}{$\mathbf{A B}_{2}$} & \multicolumn{2}{|c|}{$\mathrm{AB}_{3}$} \\
\hline $\begin{array}{l}\text { Tetraauricupride } \\
\text { CuAu P4/mmm }\end{array}$ & & $\begin{array}{c}\text { Rutile } \\
\mathrm{TiO}_{2} \quad \mathrm{P}_{2} / \mathrm{mnm}\end{array}$ & & $\begin{array}{l}\text { Auricupride } \\
\mathrm{Cu}_{3} \mathrm{Au} \quad \operatorname{Pm} \overline{3} \mathrm{~m}\end{array}$ & \\
\hline 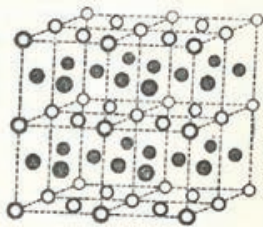 & & & & 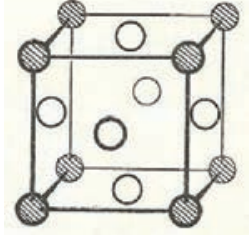 & \\
\hline $\begin{array}{c}\text { Hongshiite } \mathrm{PtCu} \\
\text { R32... }\end{array}$ & & $\begin{array}{l}\text { Scrutinyite } \\
\mathrm{PbO}_{2} \text { Pcan }\end{array}$ & $\begin{array}{c}\text { Anatase } \\
\mathrm{TiO}_{2} \quad \text { I } 4_{1} / \text { amd }\end{array}$ & $\begin{array}{c}\text { Dyscrasite } \\
\mathrm{Ag}_{3} \mathrm{Sb} \quad \mathrm{Pmm} 2\end{array}$ & \\
\hline $\begin{array}{l}\text { Nickeline } \\
\text { NiAs } \quad \mathrm{P}_{3} / \mathrm{mmc}\end{array}$ & $\begin{array}{l}\text { Halite } \\
\mathrm{NaCl} \quad \mathrm{Fm} \overline{3} \mathrm{~m}\end{array}$ & $\begin{array}{l}\text { Ramsdellite } \\
\mathrm{MnO}_{2} \quad \text { Pbnm }\end{array}$ & & $\begin{array}{l}\text { Molysite } \\
\mathrm{FeCl}_{3} \mathrm{R} \overline{3}\end{array}$ & $\begin{array}{c}\text { Molybdite } \\
\mathrm{MoO}_{3} \text { Pbnm }\end{array}$ \\
\hline & & & & $\mathbf{A}$ & \\
\hline $\begin{array}{l}\text { Wurtzite-2H } \\
\text { ZnS } \mathrm{P}_{3} \mathrm{mc}\end{array}$ & 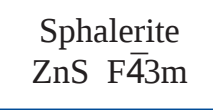 & $\begin{array}{c}\text { Melonite } \\
\mathrm{NiTe}_{2} \quad \mathrm{P} 3 \overline{\mathrm{m}} 1\end{array}$ & $\begin{array}{l}\text { Chloromagnesite } \\
\mathrm{MgCl}_{2} \quad \mathrm{R} 3 \mathrm{~m}\end{array}$ & $\begin{array}{c}\text { Roaldite } \\
\mathrm{N}(\mathrm{Fe}, \mathrm{Ni})_{4} \operatorname{Pm} \overline{\mathrm{m}}\end{array}$ & \\
\hline & 12 & 8 & 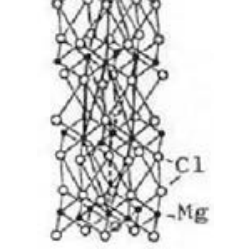 & & \\
\hline
\end{tabular}

Figura 1 - Representação convencional de algumas estruturas cristalinas de compostos de fórmulas gerais $\mathrm{AB}, \mathrm{AB}_{2}, \mathrm{AB}_{3}$ e $\mathrm{AB}_{4}$. $\mathrm{A}$ maioria das figuras aqui representadas são do livro "Structural Inorganic Chemistry", de A. F. Wells, 1950, reproduzidas com a autorização da Oxford University Press (www.oup.com) 


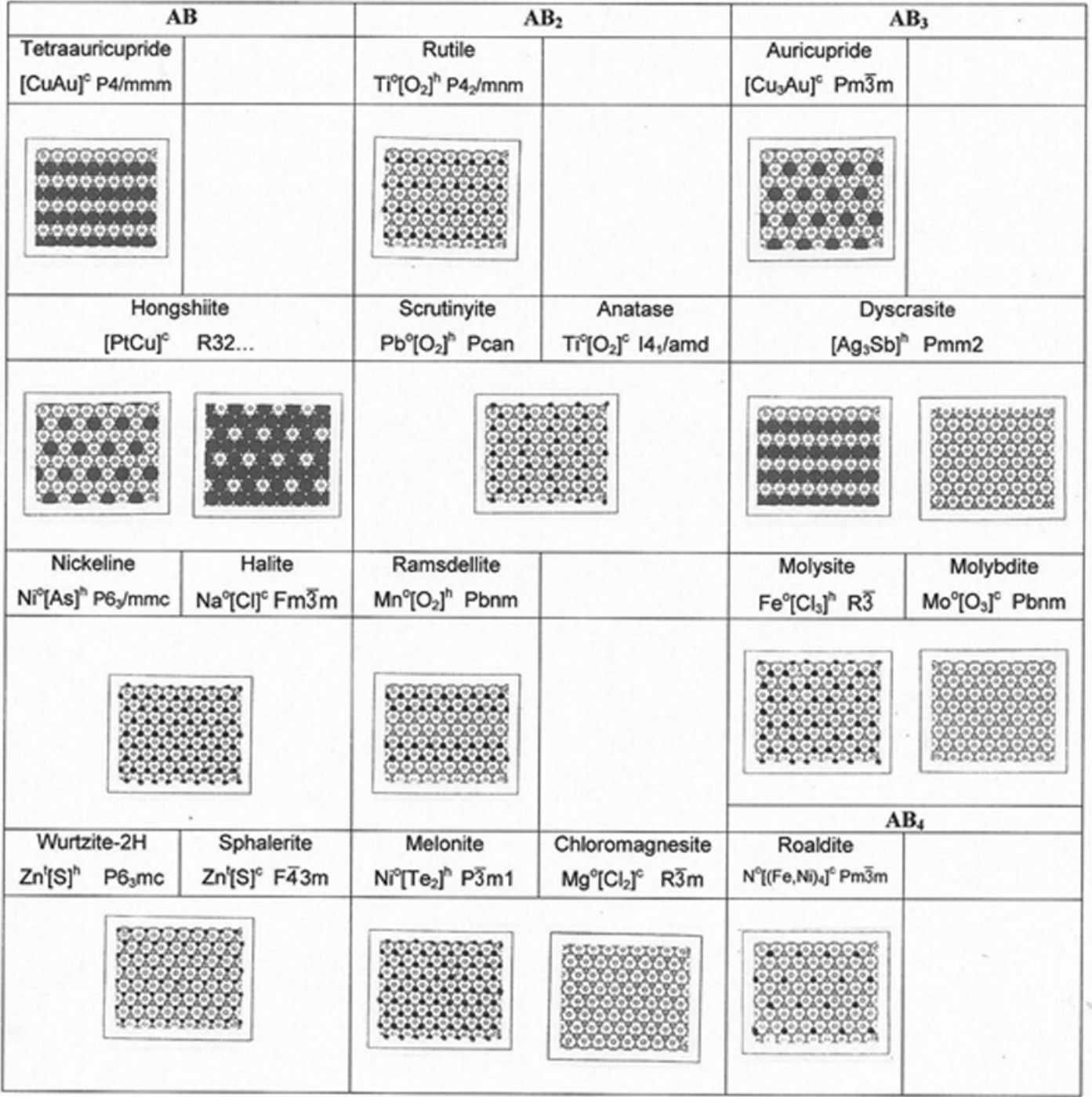

Figura 2 - Representação das estruturas cristalinas de alguns compostos de fórmulas gerais $\mathrm{AB}, \mathrm{AB}_{2}, \mathrm{AB}_{3}$ e $\mathrm{AB}_{4}$ baseadas no empilhamento denso dos átomos maiores, com os átomos mais pequenos ocupando os interstícios octaédricos ou tetraédricos, usando modelos condensados. Quando só uma camada está representada isso significa que as camadas são todas iguais, se estão representadas duas camadas significa que essas camadas se sobrepõem alternadamente. As fórmulas estruturais contêm as características das estruturas, colocando-se primeiro os átomos intersticiais com a coordenação indicada como expoente ( $o$ se octaédrica e $t$ se tetraédrica), e a seguir os átomos maiores de empilhamento denso dentro de parênteses rectos, com a indicação do tipo de empilhamento, se cúbico ou hexagonal, também como expoente (c para cúbico e $h$ para hexagonal). A simplicidade destas estruturas é evidente.

\section{REFERÊNCIAS}

[1] P. Dirac, citado por H. Corbi e K. Helge em "Paul Dirac et la Beauté de la Physique”, revista "Pour la Science”, nº 189, Julho de 1993, p.78

[2] A. Einstein, citado por Rómulo de Carvalho em "O «saber» e o «compreender»”, revista “Ciência”, 1, no 2 (1949) 37

[3] J. Lima-de-Faria, Z. Kristallogr., 122, 5/6 (1965) 346-358

[4] P.B. Moore, Aust. J. Chem., 45 (1992) 1335-1354

[5] P.B. Moore, (private communication) (1995)
[6] J. Lima-de-Faria, Eur. J. Mineral., 24 (2011) 163-169

[7] P. Nigli, “Grundlagen der Stereochemie”, Verlag Birkhäuser, Basel, 1945

French translation,” Les bases de la Stéreochimie”, Dunod, Paris, 1952

[8] F. Machatschki, Monatsh. Chem, 77 (1947) 333-342

[9] J. Lima-de-Faria, Z. Kristallogr., 122, 5/6 (1965) 359-374

[10] J. Lima-de-Faria e M.O. Figueiredo, J. Solid State Chem., 16 (1976) 7-20

[11] J. Lima-de-Faria, E. Hellner, F. Libau, E. Makovicky, e E. Parthé, Acta Crystallogr., A46 (1990) 1-11

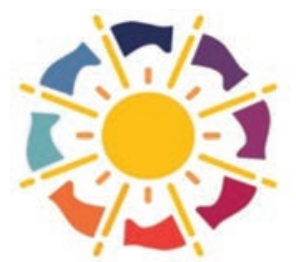

ANO INTERNACIONAL DA LUZ 2015 - CALL FOR PAPERS

Envie os seus manuscritos para bquimica@quimica.uminho.pt 